Published in final edited form as:

Infect Control Hosp Epidemiol. 2018 July ; 39(7): 882-884. doi:10.1017/ice.2018.81.

\title{
Association of an Active Surveillance and Decolonization Program on Incidence of Clinical Cultures Growing Staphylococcus aureus in the Neonatal Intensive Care Unit
}

\author{
Annie Voskertchian, MPH ${ }^{1}$, Ibukunoluwa Akinboyo, MD ${ }^{1}$, Elizabeth Colantuoni, $\mathrm{PhD}^{3}$, Julia \\ Johnson, MD², and Aaron M. Milstone, MD, MHS ${ }^{1}$ \\ ${ }^{1}$ Department of Pediatrics, Division of Infectious Disease, Johns Hopkins University, Baltimore, \\ Maryland, USA \\ ${ }^{2}$ Department of Pediatrics, Division of Neonatology, Johns Hopkins University, Baltimore, \\ Maryland, USA \\ ${ }^{3}$ Department of Biostatistics, Johns Hopkins Bloomberg School of Public Health, Baltimore, \\ Maryland, USA
}

\section{INTRODUCTION}

Staphylococcus aureus ( $S$. aureus) remains a leading cause of hospital-acquired infections (HAIs) in neonates. ${ }^{1}$ Some neonatal intensive care units (NICUs) use active surveillance cultures (ASC) and decolonization to prevent methicillin-resistant $S$. aureus (MRSA) transmission and infections. ${ }^{2}$ However, methicillin-susceptible $S$. aureus (MSSA) infections occur more frequently and have similar mortality in neonates. ${ }^{3}$

In The Johns Hopkins' NICU, prior to April 2013, neonates were screened for MRSA colonization and carriers were decolonized. ${ }^{4}$ In April 2013, the program expanded to include MSSA screening and decolonization. Previously, we showed that after implementation of MSSA ASC and targeted decolonization, $S$. aureus clinical cultures and infections decreased. ${ }^{4}$ Our objective was to assess whether the reduction was sustained over 3-years.

\section{METHODS}

Using the Johns Hopkins Pathology information system, we retrospectively identified neonates admitted to the NICU between April 1, 2011 and June 30, 2016. S. aureus-positive clinical cultures were defined as non-surveillance cultures growing $S$. aureus. Cultures from the same patient were considered unique events if collected from the same body site at least 30 days apart or from different body sites at least 14 days apart. NICU-attributable was defined as clinical cultures obtained more than two days after unit admission. Neonates were

\footnotetext{
Corresponding author: Aaron M. Milstone, MD MHS, 200 N. Wolfe St, Baltimore MD 21287, amilsto1@jhmi.edu. Previous presentation/abstract: Voskertchian, A., Akinboyo, I., Johnson, J., Colantuoni, E., Sick-Samuels, A., Aucott, S.W., Milstone, A.M. Association of Active Surveillance and Decolonization Program on Incidence of Clinical Cultures Growing Staphylococcus aureus in the Neonatal Intensive Care Unit. Poster presented at: IDWeek. 2017, Oct 4-8; San Diego, CA.

Potential conflicts of interest: A.M. and J.J. report grant support from Sage Products, LLC. All other authors report no conflicts of interest.
} 
considered to have a bloodstream infection (BSI) if blood cultures grew $S$. aureus. Incidence rates for NICU-attributable $S$. aureus clinical cultures and BSIs and $95 \%$ confidence intervals (CI) were calculated for the pre- and post-intervention periods and compared using two-sample Poisson tests. Interrupted time series models were fit to the log-transformed quarterly incidence rates to quantify the immediate impact of the program, and the relative change in incidence rates per quarter during the pre and post-intervention period. ${ }^{4}$ This study was approved by the Institutional Review Board.

\section{RESULTS}

During the 24 months before implementation (29,200 patient-days) and 39 months postimplementation (47,135 patient-days), there were 74 and 68 NICU-attributable $S$. aureus clinical cultures, respectively. There were 116 unique patients with 142 S. aureus cultures, of which $131(92 \%)$ were MSSA and $11(8 \%)$ were MRSA. Sources for the 142 isolates included $84(59.2 \%)$ respiratory, 20 (14\%) blood, 10 (7.0\%) conjunctiva, 11 (7.7\%) wound, 7 (4.9\%) other, 5 urine (3.5\%), 3 abscess (2.1\%), and 2 cerebral spinal fluid (1.4\%). Postintervention, 1847 neonates were screened for $S$. aureus colonization as part of the ASC and decolonization program. Of the 333 colonized, 243 were treated with mupirocin.

Overall, a $43 \%$ reduction in the incidence rate of $S$. aureus clinical isolates occurred when comparing the post- to the pre-intervention period (IRR: 0.57, 95\% CI: 0.40-0.80) (Figure 1a). Prior to the intervention, the incidence rate of $S$. aureus clinical cultures was estimated to increase at a non-significant rate of $14 \%$ per quarter (IRR: 1.14, 95\% CI: $0.95-1.38$ ). In the quarter following introduction of MSSA to the ASC program, there was an immediate 65\% decrease (IRR: $0.35,95 \%$ CI: $0.15-0.82$ ) and thereafter an estimated $2.0 \%$ quarterly decrease in the incidence of NICU-attributable $S$. aureus clinical cultures (IRR: 0.98, 95\% CI: $0.92-1.05)$. The rate at which the incidence rates changed over time during the pre- and post-intervention periods did not differ statistically (estimated relative quarterly rate of change: 0.86 , p-value: 0.12 ).

Prior to the intervention, there was no change in the incidence rate of BSIs (IRR: 1.00, 95\% CI: 0.78-1.29). After implementation, there were non-statistically significant reductions in the overall incidence rate of $S$. aureus BSIs (IRR: 0.50, 95\% CI 0.18-1.34) (Figure 1b), the immediate change in rate of $S$. aureus BSIs (IRR: $0.73,95 \%$ CI: $0.20-2.58$ ) and the quarterly incidence rate of $S$. aureus BSIs (IRR: 0.97, 95\% CI: 0.92-1.03).

With an average of 5.5 BSIs per year in the pre-intervention period, 18 BSIs were expected to occur in the post-intervention period, yet we observed 9. In the setting of $\geq 70 \%$ compliance with the decolonization protocol, $50 \%$ fewer infections occurred than expected, suggesting 27 neonates were treated to prevent $1 \mathrm{BSI}$.

\section{DISCUSSION}

Our data suggest an active MSSA screening and decolonization program in the NICU can lead to a sustained reduction (overall $43 \%$ decrease) in the incidence of clinical $S$. aureus isolates. 
Prior studies have found ASC and decolonization, in conjunction with other infection control measures, can reduce MRSA colonization and infection. ${ }^{2,5}$ Burden of MSSA infections exceed MRSA infections in the NICU, ${ }^{3}$ yet, few studies have examined the impact of MSSA ASC and decolonization. Recently Wisgrill et af 6 reported promising results of an MSSA surveillance and decolonization program that led to a 50\% reduction of MSSA-attributable infections in very low-birth weight infants. Our study reports similar findings, and, we included all neonates admitted to the NICU to reflect the impact on overall burden of $S$. aureus HAIs.

Efficacy, cost-effectiveness and safety influence the decision to perform ASC and decolonization. While there are limited neonatal data, reports from adult populations suggest active surveillance, targeted decolonization, and at times universal decolonization are cost effective when compared to other prevention methods. ${ }^{7}$ Possible unintended consequences of decolonization include replacing $S$. aureus with more virulent pathogens. However, in a multi-center NICU study examining MRSA decolonization, patients treated with mupirocin did not show increased risk of novel Gram negative and fungal infections. ${ }^{8}$ Emerging resistance to mupirocin must also be considered with widespread use, but recent $S$. aureus ASC and decolonization programs have not found an increase in resistance to mupirocin. $4-6,9$

Incorporating MSSA screening into a NICU's infection control protocol may be an important step to reduce $S$. aureus infections in this vulnerable neonatal population.

\section{ACKNOWLEDGEMENTS}

The authors would like to thank Qumars Roshanian at the Johns Hopkins Pathology Data Services for his assistance in extracting microbiology culture data, and Avi Gadala in the Johns Hopkins Hospital Department of Hospital Epidemiology and Infection Control for his assistance with data management.

Financial support: This study was partially funded by the Agency for Healthcare Research and Quality (AHRQ) 1R01HS022872.

\section{REFERENCES}

1. Verstraete E, Boelens J, De Coen K, et al. Healthcare-associated bloodstream infections in a neonatal intensive care unit over a 20-year period (1992-2011): trends in incidence, pathogens, and mortality. Infection control and hospital epidemiology 2014;35:511-518. [PubMed: 24709719]

2. Huang YC, Lien RI, Su LH, Chou YH , Lin TY . Successful control of methicillin-resistant Staphylococcus aureus in endemic neonatal intensive care units--a 7-year campaign. PloS one 2011;6:e23001. [PubMed: 21857979]

3. Ericson JE, Popoola VO, Smith PB , et al. Burden of Invasive Staphylococcus aureus Infections in Hospitalized Infants. JAMA pediatrics 2015;169:1105-1111. [PubMed: 26502073]

4. Popoola VO, Colantuoni E, Suwantarat N, et al. Active Surveillance Cultures and Decolonization to Reduce Staphylococcus aureus Infections in the Neonatal Intensive Care Unit. Infect Control Hosp Epidemiol 2016;37:381-387. [PubMed: 26725699]

5. Delaney HM , Wang E, Melish M . Comprehensive strategy including prophylactic mupirocin to reduce Staphylococcus aureus colonization and infection in high-risk neonates. Journal of perinatology : official journal of the California Perinatal Association 2013;33:313-318. [PubMed: 22918547] 
6. Wisgrill L, Zizka J , Unterasinger L, et al. Active Surveillance Cultures and Targeted Decolonization Are Associated with Reduced Methicillin-Susceptible Staphylococcus aureus Infections in VLBW Infants. Neonatology 2017;112:267-273. [PubMed: 28704818]

7. Whittington MD, Atherly AJ, Curtis DJ, Lindrooth RC, Bradley CJ , Campbell JD . Recommendations for Methicillin-Resistant Staphylococcus aureus Prevention in Adult ICUs: A Cost-Effectiveness Analysis. Critical care medicine 2017;45:1304-1310. [PubMed: 28471887]

8. Pierce R , Bryant K, Elward A, Lessler J , Milstone AM . Bacterial Infections in Neonates Following Mupirocin-Based MRSA Decolonization: A Multicenter Cohort Study. Infection control and hospital epidemiology 2017;38:930-936. [PubMed: 28578731]

9. Hayden MK , Lolans K, Haffenreffer K, et al. Chlorhexidine and Mupirocin Susceptibility of Methicillin-Resistant Staphylococcus aureus Isolates in the REDUCE-MRSA Trial. Journal of clinical microbiology 2016;54:2735-2742. [PubMed: 27558180] 
A

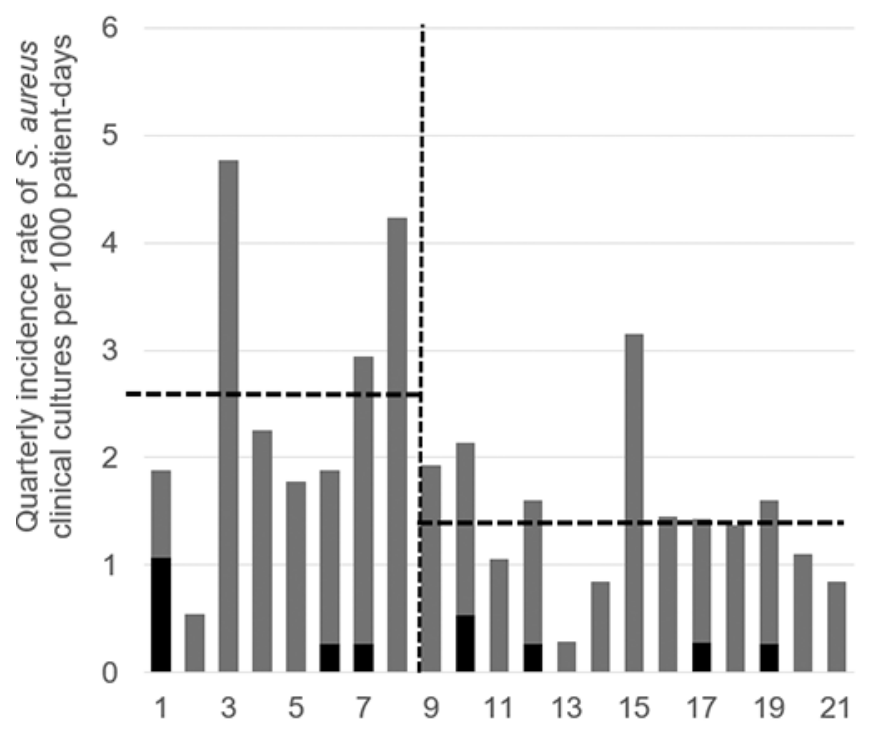

B

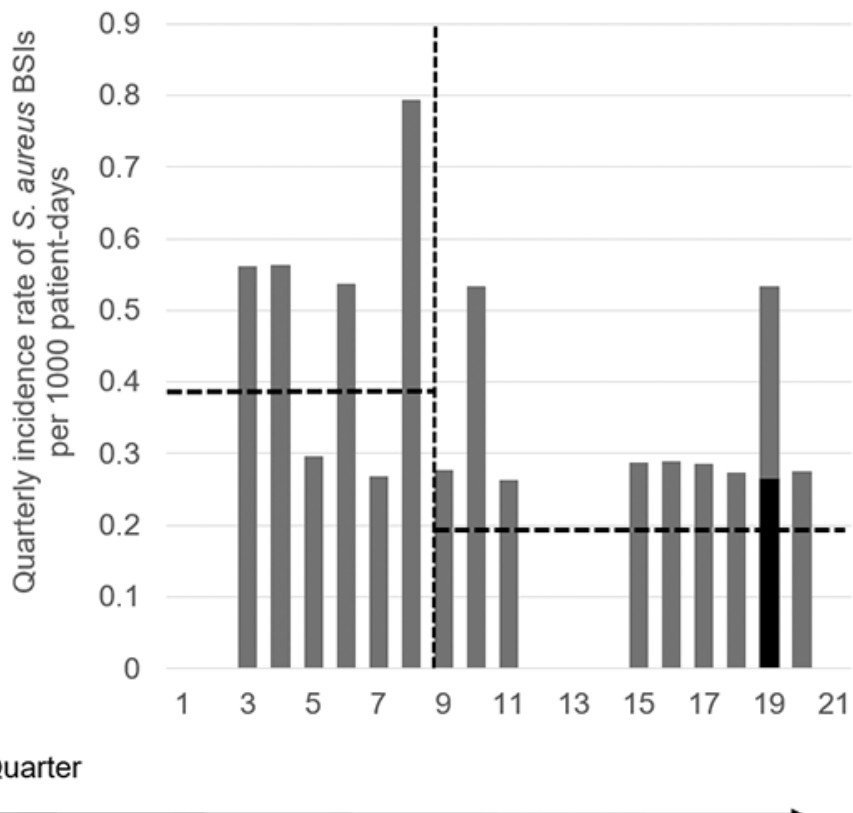

- Methicillin-resistant S. aureus = Methicillin-susceptible S. aureus

Figure:

(A) Quarterly incidence rate of Staphylococcus aureus (S. aureus) clinical cultures before (April 1, 2011-March 30, 2013) and after (April 1, 2013-June 30, 2016) implementation of a methicillin-susceptible $S$. aureus (MSSA) and methicillin-resistant $S$. aureus (MRSA) active surveillance and decolonization protocol. Dashed horizontal lines show the average incidence rate of $S$. aureus during the pre- and post-periods and the dashed vertical line represents start of intervention (Quarter 9). MRSA and MSSA are depicted in black and gray, respectively. (B) Quarterly incidence of $S$. aureus bloodstream infections (BSIs) before and after implementation of a methicillin-susceptible $S$. aureus (MSSA) and methicillinresistant $S$. aureus (MRSA) active surveillance and decolonization protocol. 